\title{
Dose-response studies of Ropivacaine in blood flow of upper extremity after supraclavicular block: a double-blind randomized controlled study
}

Ting $\mathrm{Li}^{1,2} \mathbb{D}$, Qiguang $Y \mathrm{e}^{2}$, Daozhu $\mathrm{Wu}^{3}$, Jun $\mathrm{Li}^{2}$ and Jingui $Y \mathrm{u}^{1^{*}}$

\begin{abstract}
Background: The sympathetic block of upper limb leading to increased blood flow has important clinical implication in microvascular surgery. However, little is known regarding the relationship between concentration of local anesthetic and blood flow of upper limb. The aim of this dose-response study was to determine the $\mathrm{ED}_{50}$ and $\mathrm{ED}_{95}$ of ropivacaine in blood flow after supraclavicular block (SB).

Methods: Patients undergoing upper limb surgery and supraclavicular block were randomly assigned to receive 30ml ropivacaine in concentrations of 0.125\%(A Group), 0.2\%(B Group), 0.25\%(C Group), 0.375\%(D Group), 0.5\%(E Group), or $0.75 \%$ (F Group) ( $n=13$ per group). All patients received supraclavicular block (SB). Time average maximum velocity (TAMAX), cross-sectional area (CSA) of brachial artery and skin temperatures $\left(T_{s}\right)$ were measured repeatedly at the same marked points, they were taken at baseline (before block, $\left.\mathrm{t}_{0}\right)$ and at 30min after SB $\left(\mathrm{t}_{1}\right)$. Blood flow $(B F)=T A M A X \times C S A \times 60$ sec.. Relative blood flow $(\Delta B F)=B F_{t 1} / B_{t 0}$. Success of $S B$ was assessed simultaneously. Supplementary anesthesia and other adverse events (AE) were recorded.

Results: Significant increase in TAMAX, CSA, BF and $T_{s}$ were seen in all concentration groups at $t_{1}$ comparing with $\mathrm{t}_{0}(P<0.001)$. There was an upward trend of TAMAX, CSA, BF with the increasing concentration of ropivacaine except $T_{s}$. There was no significant different of $T_{s}$ at $t_{1}$ among different concentration group. The dose-response formula of ropivacaine on $\Delta \mathrm{BF}$ was $\mathrm{Y}=1+3.188 /(1+10 \wedge((-2.451-\mathrm{X}) \times 1.730))$ and $\mathrm{ED}_{50} / \mathrm{ED}_{95}(95 \% \mathrm{Cl})$ were $0.35 / 1$. $94 \%(0.25-0.45 / 0.83-4.52)$, and $R^{2}$ (coefficient of determination) $=0.85$. $\mathrm{ED}_{50} / \mathrm{ED}_{95}(95 \% \mathrm{Cl}$ ) values of sensory block were $0.18 / 0.33 \%(0.15-0.21 / 0.27-0.51), R^{2}=0.904$.
\end{abstract}

Conclusions: The dose-response curve between SB ropivacaine and the changes of BF was determined. The $\mathrm{ED}_{50}$ / $\mathrm{ED}_{95}$ of ropivacaine of $\triangle \mathrm{BF}$ are $0.35 / 1.94 \%$ (0.25-0.45/0.83-4.52). TAMAX, CSA and BF consistently increased with ropivacaine concentration. The maximal sympathetic block needs higher concentration than that complete sensation block needs which may benefit for microvascular surgery.

Trial registration: Clinicaltrials.gov NCT02139982. Retrospectively registered (Date of registration: May, 2014).

Keywords: Brachial plexus, nerve block, Local anesthetic, Hemodynamics, Upper extremity

\footnotetext{
* Correspondence: yujingui1 109@126.com

'Department of Anesthesiology, Qilu Hospital of Shandong University, Jinan,

China

Full list of author information is available at the end of the article
} 


\section{Background}

Brachial plexus block (BPB) is widely used in regional anesthesia [1]. Its sympathetic block leads to vasodilatation and increased blood flow and skin temperature in the ipsilateral upper limb [2-4]. So this technique is especially useful for microvascular surgery such as replantation of digits. Currently, there are several literatures of regional hemodynamic changes of arteriovenous fistula after a BPB [5-7]. The role of increased blood flow after BPB is considered to be of great importance for microvascular surgery.

Recent publications have reported dose-finding studies of sensory block in brachial plexus and given the $\mathrm{ED}_{95}$ or $\mathrm{ED}_{50}$ dose $[8,9]$. However, there is no literature about dose-finding studies of sympathetic block in brachial plexus. Little is known regarding the relationship between concentration of local anesthetic and blood flow of upper extremity. The aim of this randomized, double-blind, concentration-response study was to determine the $\mathrm{ED}_{50}$ and $\mathrm{ED}_{95}$ of ropivacaine in blood flow of upper extremity after supraclavicular block.

\section{Methods}

This double-blind randomized controlled, dose-response study was conducted in China. Study approval was obtained from the Institutional Review Board (IRB) of the Second Affiliated Hospital and Yuying Children Hospital of Wenzhou Medical University (109 Xueyuang xi Road, Wenzhou, China, Ref 2009(002), February 2th, 2009, Prof. Qinquan Lian). This trial was registered at Clinicaltrials.gov with the identifier NCT02139982 on 28 May 2014. Informed consent was obtained from each patient. Between July 2011 and June 2012, all consecutive adults undergoing upper extremity surgery were screened for the trial. Inclusion criteria were adults ( $>18$-year-old), American Society of Anesthesiologists (ASA) physical status I-III and elective surgery. Exclusion criteria were infection at the site of needle insertion; any coagulopathy; allergy to local anesthetics; peripheral neurological disease, or peripheral vascular disease. Patients were randomly assigned to receive $30 \mathrm{ml}$ ropivacaine (Naropin, Astra-Zeneca, Sweden) in concentrations of $0.125 \%$ (A Group), $0.2 \%$ (B Group), $0.25 \%$ (C Group), $0.375 \%(\mathrm{D}$ Group), $0.5 \%$ (E Group), or $0.75 \%$ (F Group) using computer (Microsoft excel 2010) generated random number in sealed envelopes.

Each patient lay supine in a hospital bed in the recovery room with room temperature of $24{ }^{\circ} \mathrm{C}$, acclimatizing for $15 \mathrm{~min}$. All patients were performed routine monitoring (NIAP, continuous ECG, and pulse oximetry). All nerve blocks were performed by a single dedicated anesthetist. Time average maximum velocity (TAMAX), cross-sectional area (CSA) of brachial artery and skin temperatures $\left(\mathrm{T}_{\mathrm{s}}\right)$ were measured repeatedly at the same marked points at baseline (before block, $t_{0}$ ) and at $30 \mathrm{~min}$ after SB $\left(t_{1}\right)$. TAMAX is a sensitive hemodynamic index, increased with the vasodilatation and the reduction of peripheral vascular resistance. CSA is a direct index of vasodilatation. Heart rate (HR) and mean arterial pressure (MAP) were recorded simultaneously. All these measurements were performed by another investigator who does not know the concentration of ropivacaine. For patients with an ineffective block before surgery, supplementary anesthesia (conversions to general anesthesia, supplementary brachial plexus block or branch nerve block with $10-20 \mathrm{ml}$ of ropivacaine $0.5 \%$ ) was administered according to the site of surgery and anesthetist's experience. If the patient experienced any pain during his surgery, supplementary analgesia (fentanyl 1 to $2 \mu \mathrm{cg} / \mathrm{kg}$ ), sedation (midazolam 10 to $30 \mu \mathrm{cg} / \mathrm{kg}$ ), or general anesthesia was administered as required. Supplementary anesthesia and other adverse events (AE) were recorded.

\section{Supraclavicular block}

A single anesthetist with 7 years experience of ultrasoundguided nerve blocks performed all brachial plexus blocks. The brachial plexus was visualized with a high-frequency linear ultrasound transducer (HFL 38X/13-6 MHz, Snerve TM Ultrasound System, SonoSite Inc., USA) in the supraclavicular fossa. After disinfection and infiltrating with $1 \%$ lidocaine, by in-line technique, nerve stimulation needle (Stimuplex ${ }^{\ominus}$ D, 22G, 50 mm; B.Braun Melsungen AG, Germany) was inserted and advanced among the divisions of brachial plexus with electric impulses $(2 \mathrm{~Hz}$, $0.5 \mathrm{~mA}, 0.1 \mathrm{~ms}$ ) of nerve stimulator (Stimuplex ${ }^{\oplus}$ HNS 11 Peripheral Nerve Stimulator, B.Braun Melsungen AG, Germany). If visible contraction of the innervated muscle was elicited, the needle was withdrawn slowly until the corresponding muscle contraction disappeared to avoid intrafascicular puncture [10]. The local anesthetic was injected at three locations: adjacent to the superficial divisions of the plexus, adjacent to the middle divisions and adjacent to inferior divisions. The spread of local anesthetic was observed to ensure the quality of the performance of BPB. The proportion of the volume injected in each area was at the discretion of the expert operator according to the spread of local anesthetic. This operator remained blind to the concentration of ropivacaine.

\section{Block assessment}

Another investigator, who was not present during the conduct of the SB and was completely blinded to the concentration of ropivacaine used, assessed each blockade. The subjects did not know the concentration of local anesthetic used either. The efficacy of the block was assessed by pinprick sensation (22 G needle) and compared with the opposite forearm/hand and 
recorded as sensation, hypoesthesia or no sensation at 30 min after the SB. Success of SB was defined as the absence of sensation in all innervation areas of four nerves (musculocutaneous, ulnar, radial, and median nerves) $30 \mathrm{~min}$ after the $\mathrm{SB}$ and no pain during the surgery.

\section{Measurement of hemodynamic parameters}

Patient's ipsilateral forearm was in supination. The brachial artery was located in $2 \mathrm{~cm}$ proximal to the antecubital fossa. Specific points were located with skin marker to provide consistency with all measurements taken. Hemodynamic Parameters were measured by Pulsed-wave Doppler (PWD) ultrasound (DC-6, Mindray Medical International Limited, China) with a 7 L4 linear array transducer (frequency $10 \mathrm{MHz}$ ). The probe was parallel the long axis of the arm without undue pressure on the artery during the PWD measurements. The volume gate was positioned in the center of the arterial lumen, and the size of the gate was $1 / 3 \mathrm{~lm}$ of the artery. The angle of insonation was adjusted and maintained at 50-60 degrees. Once a desired PWD spectral waveform was achieved, the arterial TAMAX was recorded. (Fig. 1). CSA of the artery was assessed with Bmode imaging. Probe should be as perpendicular as possible to the long axis of the artery to obtain as round an arterial section as possible. The image at end diastole was chosen with the cine loop. The changes in arterial cross-sectional area during the cardiac cycle was not measured in this study. BF was calculated by formula: $\mathrm{BF}=\mathrm{TAMAX} \times \mathrm{CSA} \times 60$ s. Relative blood flow $(\triangle \mathrm{BF})=\mathrm{BF}_{\mathrm{t} 1} / \mathrm{BF}_{\mathrm{t} 0}$.

\section{Measurement of temperature}

Skin temperature $\left(\mathrm{T}_{\mathrm{s}}\right)$ was measured at the thenar by the rapid precise contact thermometer (DAE-905 T, ShengGao Inc., China). Specific points were located with skin marker to provide consistency of measurement.

\section{Statistical analysis}

GraphPad Prism for Windows 5.0 (GraphPad Software, Inc., San Diego, USA) was used for nonlinear regression to build dose-response of $\triangle \mathrm{BF}$. The equation of nonlinear regression is:

$$
Y=\text { Bottom }+\frac{(\text { Top-Bottom })}{1+10^{\left(\log E C_{F}-X\right) \times \text { Hillslope }}}
$$

Where $X$ is the logarithm of concentration of ropivacaine, $Y$ is the relative blow flow $\triangle \mathrm{BF}, F$ is a constant value between 0 and 100, Top is the $\mathrm{Y}$ value at the top plateau, Bottom is the Y value at the bottom plateau, is the $\mathrm{X}$ value when the response is halfway between Bottom and Top and HillSlope is the steepness of the curve.

Personal information, surgical details, MAP, HR, hemodynamics and $T_{s}$ were collected and the data were presented as mean (SD) or numbers as appropriate. Paired-Samples $\mathrm{T}$ test was applied to compare hemodynamic parameters and skin temperature before and after brachial plexus block, Kruskal-Wallis $\mathrm{H}$ nonparametric test was applied to compare these variables among different concentration groups. Analysis of variance trend test and Jonckheere's trend test were performed to detect the trend in changes of hemodynamic parameters and skin temperature as the concentration

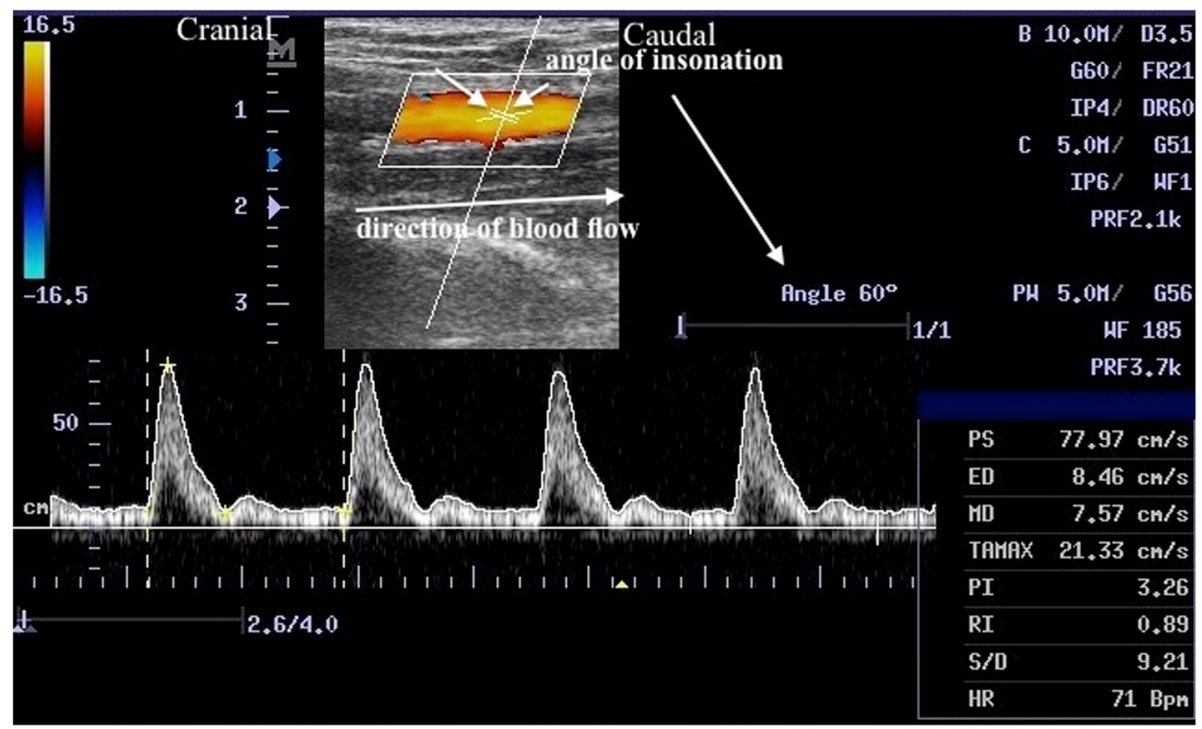

Fig. 1 PWD Ultrasound of the brachial artery after brachial plexus block. PS: peak systolic velocity (cm/s.). ED: end-diastolic velocity ( $\mathrm{cm} / \mathrm{s}$.). TAMAX: time average maximum velocity $(\mathrm{cm} / \mathrm{s}$.) 
increased. Probit regression was used to calculate the $E D_{50}$ and $E_{95}$ of ropivacaine on sensory block. For all tests, significance was defined as a $p$ value $<0.05$. Statistical analysis was conducted using SPSS for Windows 16.0 (SPSS Inc., Chicago, USA).

\section{Results}

The subjects approached are summarized using a CONSORT flow diagram (Fig. 2). All patients were undergoing elective surgery (24 removal of metalwork from ulnar/radial, 18 fixation of fractured ulnar/radial, 12 fixation of fractured metacarpal, 11 fractured phalanx, 6 amputation of finger and 7 surgical removal of soft tissue mass). There was no significant change detected in the heart rate and blood pressure after the SB across the six groups (Table 1).

\section{Measurements of hemodynamic parameters and skin temperature}

Changes in TAMAX, CSA and BF of the brachial artery and $T_{s}$ were summarized in Table 2. Significant increases in TAMAX, CSA, BF and $\mathrm{T}_{\mathrm{s}}$ were seen in all concentration groups at $t_{1}$ comparing with $t_{0}(P<0.001)$. There were not significant difference in TAMAX, CSA, BF and $\mathrm{T}_{\mathrm{s}}$ at $\mathrm{t}_{0}$ among different concentration groups $(P>0.05)$. TAMAX, CSA and BF consistently increased with concentration $(P<0.05)$ while Ts increased monotonically from the $0.125 \%$ group to the $0.5 \%$ group $(P<0.05)$ then remained as the concentration reached $0.75 \%$. The dose-response curve and data of ropivacaine on $\triangle \mathrm{BF}$ was shown in Fig. 3. The dose-response formula was

$$
Y=1+\frac{3.188}{1+10^{(-2.451-x)} \times 1.730}
$$

$\mathrm{ED}_{50} / \mathrm{ED}_{95}(95 \% \mathrm{CI})$ values of $\triangle \mathrm{BF}$ were $0.35 /$ $1.94 \%(0.25-0.45 / 0.83-4.52)$, and $R^{2}$ (coefficient of determination) $=0.85$.

\section{Assessment of sensory block}

The successful rates of sensory block in different concentration groups were: 1/13(A group), 9/13(B group), 11/ 13(C group), 12/13(D group), 13/13(E group), 13/13(F group) respectively. Fig. 4 showed the predicted concentration-response curves of ropivacaine on sensory block

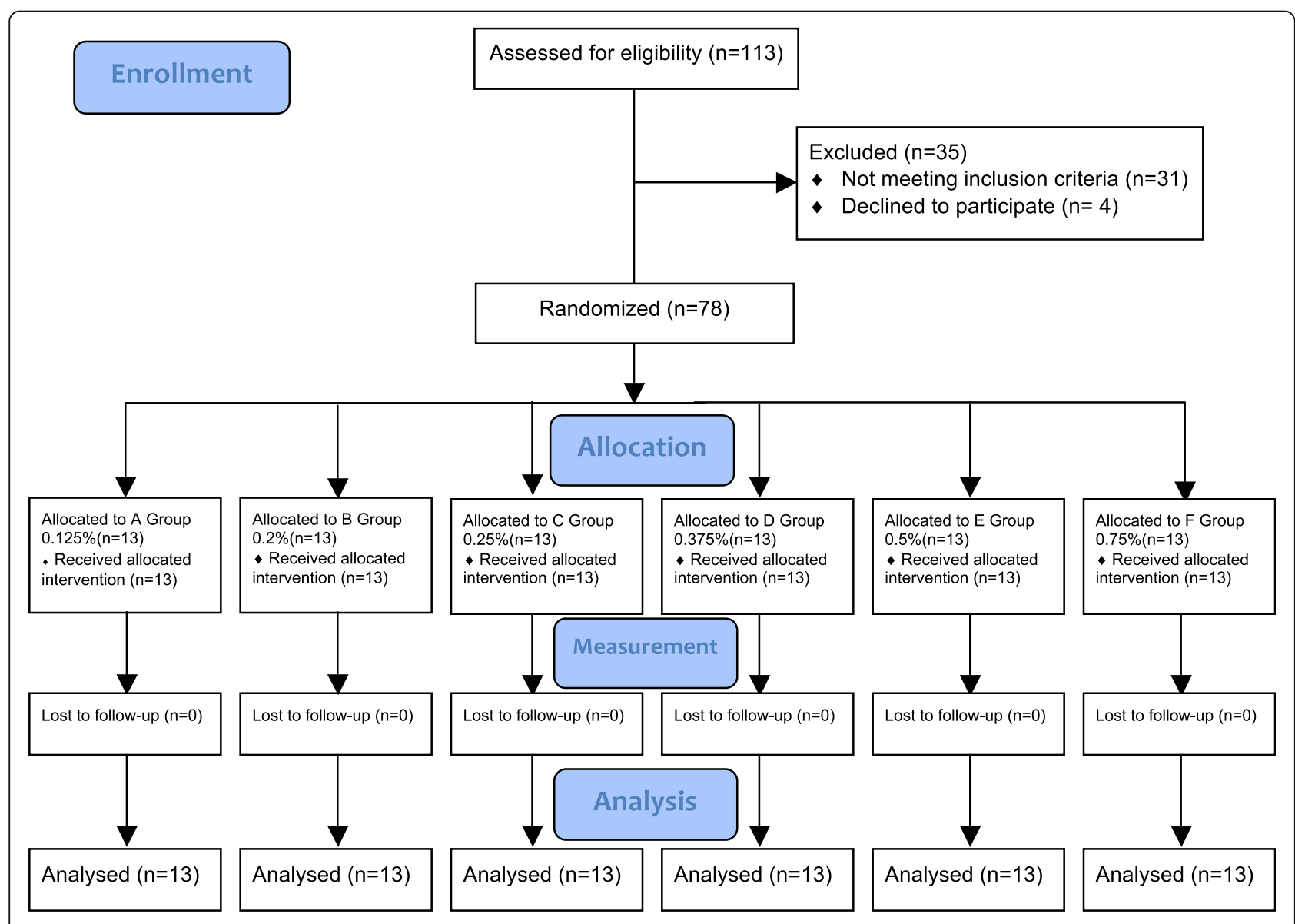

Fig. 2 The CONSORT flow diagram for randomized controlled trials 
Table 1 Patients characteristics and changes of MAP and HR in different concentration groups at baseline $\left(\mathrm{t}_{0}\right)$ and 30 min after brachial plexus block $\left(\mathrm{t}_{1}\right)$

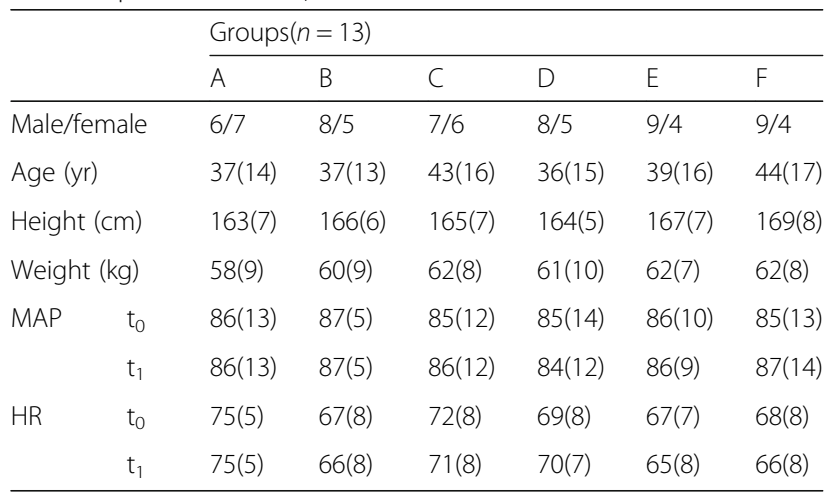

Values are number or mean (SD)

MAP Mean Arterial Pressure, HR Heart Rate

of $\mathrm{SB} . \mathrm{ED}_{50} / \mathrm{ED}_{95}(95 \% \mathrm{CI})$ values were $0.18 / 0.33 \%(0.15-$ $0.21 / 0.27-0.51)$ and $R^{2}=0.904$.

\section{Adverse event}

The rate of supplementary anesthesia in different concentration groups were: 11/13(A group), 2/13(B group), 1/13(C group), 0/13( $\mathrm{D}$ group), 0/13(E group), 0/13(F group). Total 5 patients were administered supplementary analgesia and sedation in A-D group. Two (2/13) and (1/13) of Horner syndrome occurred in $\mathrm{F}$ group and D group respectively. One (1/13) patient presented hoarseness in F group. All these AE was recovered. No dyspnea or symptoms of local anesthetic toxicity was reported in all group.

\section{Discussion}

In this study, changes in TAMAX, CSA, BF were seen in all concentration groups with an upward trend as the concentration increased. Compared to baseline, BF increased from about 1.5 -fold to 3.5 -fold with the concentration of ropivacine increased. To minimize the bias of individual different, we used $\triangle B F$ to build the dose-response curve. The estimated $\mathrm{ED}_{50} / \mathrm{ED}_{95}$ of $\triangle \mathrm{BF}$ were $0.35 /$ $1.94 \%$. It means that using these concentrations will get $50 / 95 \%$ the extreme blood flow. Simultaneously the sensory block was assessed to build dose-response curves by probit analysis. $\mathrm{ED}_{50} / \mathrm{ED}_{95}$ values of sensory block were $0.18 / 0.33 \%$ which were similar to Fredrickson's study [11]. It means these concentrations make $50 / 90 \%$ patients lose sensation. Obviously, the $\mathrm{ED}_{50} /$ $\mathrm{ED}_{95}$ of $\triangle \mathrm{BF}$ are higher than $\mathrm{ED}_{50} / \mathrm{ED}_{95}$ of sensory block respectively. The $\mathrm{ED}_{95}$ of sensory block is equivalent to the $\mathrm{ED}_{50}$ of $\triangle \mathrm{BF}$. This result indicates that sensory block is achieved by lower concentration of ropivacaine than complete sympathetic block. High concentration of ropivacaine is required to maximize sympathetic block. We may administer higher concentration of local anesthetic than normal postoperative analgesia concentration $(0.2 \%)$ to get more vasodilatation and reduction of peripheral vascular resistance which may benefit for microvascular surgery. However, the extrapolating $\mathrm{ED}_{95}$ of $\triangle \mathrm{BF}$ is higher than the maximum trial concentration (ropivacaine $0.75 \%$ ), its value may not be reliable. Additionally, both dose-response curves have high $\mathrm{R}^{2}$ value, means they are with well fitted.

There is not a consistent upward trend of $\mathrm{T}_{\mathrm{s}}$ following the increasing concentration. So we didn't build the doseresponse curve of $T_{s}$. Though the rising $T_{s}$ is a result of the increase of skin blood flow, $\mathrm{T}_{\mathrm{s}}$ is affected by many factors. Thus we believe that the blood flow is a more sensitive index for intensity of sympathetic block in upper limb than $\mathrm{T}_{\mathrm{s}}$. And also $\mathrm{T}_{\mathrm{s}}$ rises significantly with very low successful rate of sensory block in the lowest concentration $(0.125 \%)$. It agrees with Hermanns's study that $\mathrm{T}_{\mathrm{s}}$ is of limited clinical value to predict the success of sensor block during interscalene blockade [12].

Table 2 Hemodynamic parameters of brachial artery and temperature of skin in different concentration groups at baseline ( $\left.\mathrm{t}_{0}\right)$ and 30 min after brachial plexus block $\left(t_{1}\right)$

\begin{tabular}{|c|c|c|c|c|c|c|c|}
\hline & & \multicolumn{6}{|c|}{ Groups $(n=13)$} \\
\hline & & A & $B$ & C & $\mathrm{D}$ & $E$ & $\mathrm{~F}$ \\
\hline \multirow[t]{2}{*}{ TAMAX (cm/s) } & $t_{0}$ & 23.1(5.6) & $20.9(5.9)$ & 19.6(5.6) & $19.1(6.0)$ & $18.7(8.4)$ & $19.1(7.0)$ \\
\hline & $\mathrm{t}_{1}$ & $29.7(7.5)^{*}$ & $33.6(8.1)^{*}$ & $35.5(8.1)^{*}$ & $39.1(10.6)^{*}$ & $44.8(18.8)^{*}$ & $49.3(19.9)^{*}$ \\
\hline \multirow[t]{2}{*}{$\operatorname{CSA}\left(\mathrm{cm}^{2}\right)$} & $t_{0}$ & $0.126(0.025)$ & $0.140(0.056)$ & $0.133(0.031)$ & $0.131(0.035)$ & $0.114(0.028)$ & $0.114(0.038)$ \\
\hline & $\mathrm{t}_{1}$ & $0.145(0.032)^{*}$ & $0.159(0.062)^{*}$ & $0.155(0.038)^{*}$ & $0.162(0.042)^{*}$ & $0.149(0.038)^{*}$ & $0.155(0.055)^{*}$ \\
\hline \multirow[t]{2}{*}{$B F(\mathrm{ml} / \mathrm{s})$} & $t_{0}$ & $178.2(62.5)$ & 175.9(93.6) & 158.2(65.0) & 148.6(59.8) & $127.6(61.7)$ & 135.5(71.3) \\
\hline & $\mathrm{t}_{1}$ & $259.3(87.7)^{*}$ & $320.3(152.0)^{*}$ & $330.4(118.4)^{*}$ & $378.7(143.9)^{*}$ & $399.7(190.9)^{*}$ & $465.2(240.6)^{*}$ \\
\hline \multirow[t]{2}{*}{$\mathrm{Ts}\left({ }^{\circ} \mathrm{C}\right)$} & $t_{0}$ & $31.0(1.4)$ & 29.6(1.1) & $30.2(1.4)$ & 29.5(1.3) & 29.5(1.1) & 29.8(0.8) \\
\hline & $t_{1}$ & $32.2(1.2)^{*}$ & $31.5(0.9)^{*}$ & $32.5(1.2)^{*}$ & $31.8(1.3)^{*}$ & $31.9(1.2)^{*}$ & $32.2(1.2)^{*}$ \\
\hline
\end{tabular}

Mean and SD values rounded to 2 decimal places

$P$ values are results of 2 -tailed, paired student $t$ tests. ${ }^{*} P<0.01$ compared with $t_{0}$

TAMAX time average maximum velocity $(\mathrm{cm} / \mathrm{s}), C S A$ cross section area, BF blood flow, Ts skin temperature 


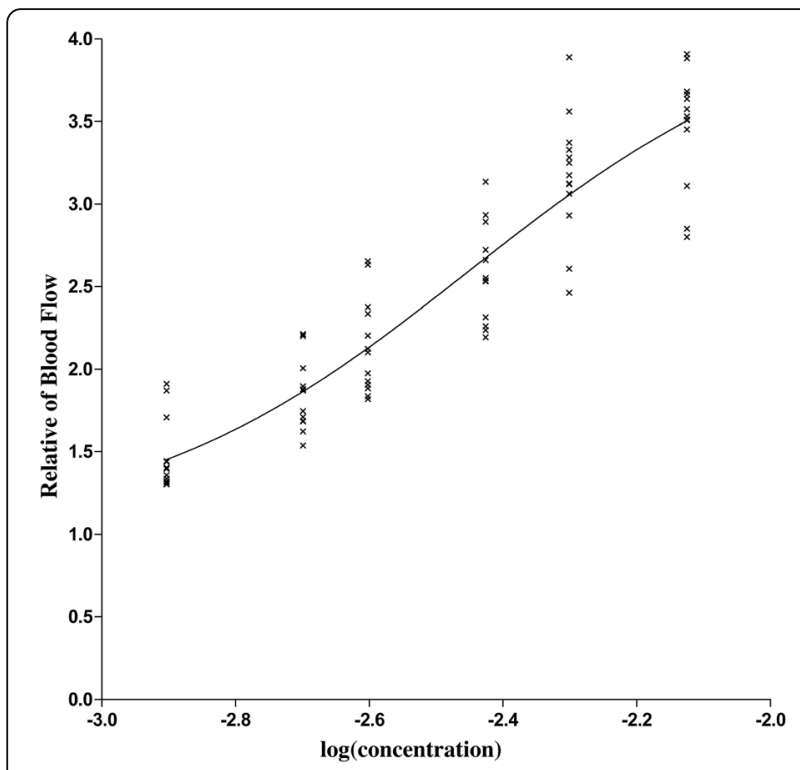

Fig. 3 The dose-response curve of value of $\triangle \mathrm{BF}(\mathrm{BFt} 1 / \mathrm{BFt} 0)$ against the log (concentration) of ropivacine and scattergram for supraclavicular block

Bigeleisen's [10] study found if stimulation threshold was greater than $0.5 \mathrm{~mA}$, the possibility of intrafascicular puncture was very little. In this study, we use ultrasound combined with nerve stimulate technique to block brachial plexus, and assure no visible contraction of the innervated muscle was elicited at $0.5 \mathrm{~mA}$ electric impulses to avoid intrafascicular puncture and to make the effect of block steady. we have not find an abnormal rapid blockade in all patients in fact.

A tightly controlled laboratory study in animals showed that the intensity of nerve blockade was primarily

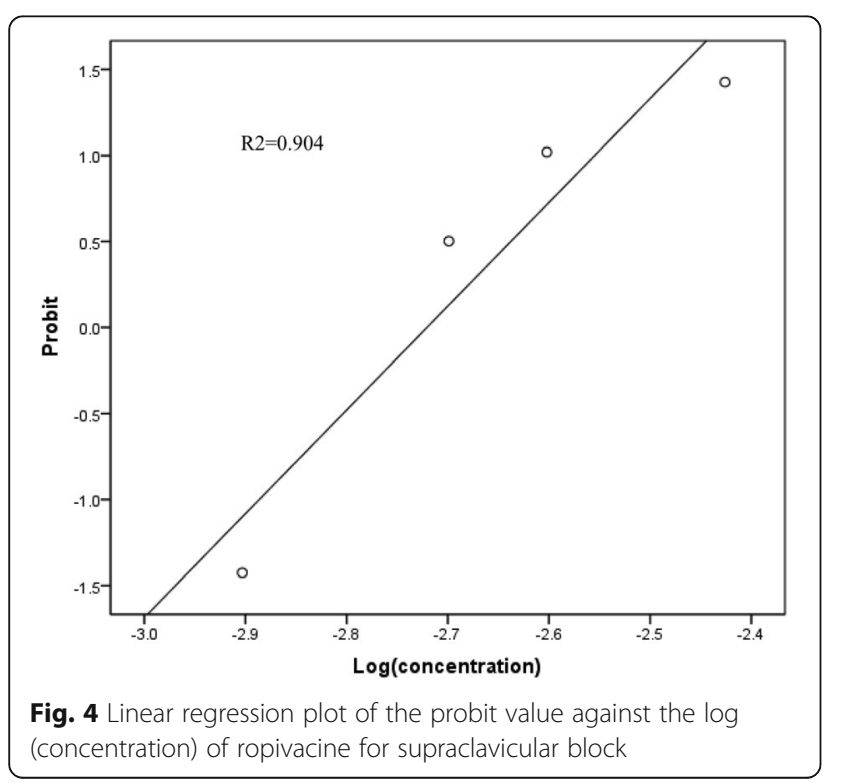

determined by local anesthetic concentration [13]. So, this study was designed to build dose-response curve with the fixed volume of local anesthetic. Kant's [8] study estimated that the $\mathrm{ED}_{95}$ dose of $0.5 \%$ Bupivacaine for Ultrasoundguided Supraclavicular Block was $27 \mathrm{ml}$. Fredrickson's [11] study reported the $\mathrm{ED}_{95}$ dose of $0.5 \%$ ropivacaine for interscalene block was $20.5 \mathrm{ml}$. However, the $\mathrm{ED}_{95}$ of volume may be different according to different concentrations and different approaches to brachial plexus block. Michał's [11] study recommended ultrasound combined nerve stimulator guided blocks (the dual guidance technique) to reduce the risk of complications for shoulder surgery with $20 \mathrm{~mL}$ of $0.5 \%$ ropivacaine. Vandepitte [14] reported that a minimum of $7 \mathrm{ml}$ of ropivacaine $0.75 \%$ through the catheter is required for success rate and timely onset of surgical anesthesia with interscalene brachial plexus blockade. No study has reported the exact $\mathrm{ED}_{95}$ of these concentration groups in Supraclavicular Block. In our study, we used $30 \mathrm{ml}$ local anesthetic for Supraclavicular Block in all concentration groups. Lower volume would probably be enough to produce a surgical block in groups $0.375 \%-0.75 \%$ but in groups with $0.125 \%$ $0.25 \%$ ropivacaine $\mathrm{BPB}$, would probably require additional sedation, analgesia or conversion to general anesthesia. The assumed primary benefit of BPB for the patient was not only to improve the blood flow in the brachial artery and but also secondly provide a perioperative analgesia rather than perform the surgical block. Additionally, some centers like our hospital prefer combination of sedation or general with regional anesthesia over general or regional anesthesia only. [15].

Currently; PWD ultrasound is a common method to measure the Hemodynamic changes in the upper extremity [16]. Our study investigated the sympathetic effects of brachial plexus block in terms of Hemodynamics by PWD ultrasound and $\mathrm{T}_{\mathrm{s}}$. The authors took many steps to minimize measurement errors, including controlling the ambient temperature and humidity; having all measurements performed by a single investigator; using a standardized measurement of PWD ultrasound and marking the position of measurement.

However, this study suffers from some limitations. Firstly, we cannot totally avoid the intrafascicular injection that usually causes abnormal and complete block. Secondly, we are not sure that $30 \mathrm{~min}$ is enough for each concentration group to achieve maximal effect of block. Future clinical studies should focus on the optimal concentration of ropivacaine for continuous BPB with the most benefit for microvascular reconstruction surgery and minimal risk of toxicity.

\section{Conclusion}

In summary, the dose-response curve between SB ropivacaine and the changes of $\mathrm{BF}$ was determined. The $\mathrm{ED}_{50} /$ 
$\mathrm{ED}_{95}$ of ropivacaine of $\triangle \mathrm{BF}$ are $0.35 / 1.94 \%(0.25-0.45 /$ 0.83-4.52). TAMAX, CSA and BF consistently increased with ropivacaine concentration. The maximal sympathetic block needs higher concentration than that complete sensation block needs which may benefit for microvascular surgery.

\section{Abbreviations}

AE: Adverse events; ASA: American Society of Anesthesiologists physical status; BF: Blood flow; BPB: Brachial plexus block; CSA: cross-sectional area; ED: End-diastolic velocity; HR: Heart rate; IRB: Institutional Review Board; MAP: Mean arterial pressure; PS: Peak systolic velocity; PWD: Pulsed-wave Doppler; SB: Supraclavicular block; TAMAX: Time average maximum velocity; Ts: Skin temperatures; $\triangle B F$ : Relative blood flow

\section{Acknowledgements}

We would like to thank Weiyang Gao, head of Hand surgery for his designing this study from The Second Affiliated Hospital and Yuying Children Hospital of Wenzhou Medical University, Wenzhou, China.

\section{Ethical approval and consent to participate}

The study approval was obtained from the Institutional Review Board (IRB) of the Second Affiliated Hospital and Yuying Children Hospital of Wenzhou Medical University (Ref 2009(002)). Written informed consent was obtained from patient.

\section{Funding}

The clinical trial was supported by Health and Family Planning Commission of Zhejiang Province, China, Grant No. 2009A145. The funding body had no further role in the design of the study and collection, analysis, and interpretation of data and in writing the manuscript.

\section{Availability of data and materials}

The study results are available at https://clinicaltrials.gov/ct2/show/results/ NCT02139982? sect $=$ X1256. The datasets collected and analyzed of this study are available from the corresponding author upon reasonable request.

\section{Authors' contributions}

$T L$ developed and supervised the study, gathered informed consent, recruited subjects, analysed the data and performed statistical analyses, wrote the first draft, revised subsequent drafts of the manuscript. QY was involved in gathering informed consent, data collection. DW recruited subjects, analysed the data. JL developed the study and reviewed the manuscript. JY supervised the study and revised subsequent drafts of the manuscript. All authors have read and approved of the final manuscript.

\section{Consent for publication}

Not applicable.

\section{Competing interests}

The authors declare that they have no competing interests.

\section{Publisher's Note}

Springer Nature remains neutral with regard to jurisdictional claims in published maps and institutional affiliations.

\section{Author details \\ 'Department of Anesthesiology, Qilu Hospital of Shandong University, Jinan, China. ${ }^{2}$ Department of Anesthesiology, The Second Affiliated Hospital and Yuying Children Hospital of Wenzhou Medical University, Wenzhou, China. ${ }^{3}$ Ultrasonic Department, The Second Affiliated Hospital and Yuying Children Hospital of Wenzhou Medical University, Wenzhou, China.}

Received: 20 June 2017 Accepted: 16 November 2017 Published online: 02 December 2017

\section{References}

1. Kolny M, Stasiowski MJ, Zuber M, et al. Randomized, comparative study of the effectiveness of three different techniques of interscalene brachial plexus block using $0.5 \%$ ropivacaine for shoulder arthroscopy. Anaesthesiol Intensive Ther. 2017:49(1):47-52.

2. Iskandar H, Wakim N, Benard A, et al. The effects of interscalene brachial plexus block on humeral arterial blood flow: a Doppler ultrasound study. Anesth Analg. 2005;101:279Y281.

3. Shemesh D, Olsha O, Orkin D, et al. Sympathectomy-like effects of brachial plexus block in arteriovenous access surgery. Ultrasound Med Biol. 2006;32:817Y822

4. Hermanns H, Braun S, Werdehausen R, Werner A, Lipfert P, Stevens MF. Skin temperature after interscalene brachial plexus blockade. Reg Anesth Pain Med. 2007:32:481-7.

5. Sahin L, Gul R, Mizrak A, et al. Ultrasound-guided infraclavicular brachial plexus block enhances postoperative blood flow in arteriovenous fistulas. J Vasc Surg. 2011;54:749-53.

6. Hingorani AP, Ascher E, Gupta P, et al. Regional anesthesia: preferred technique for venodilatation in the creation of upper extremity arteriovenous fistulae. Vascular. 2006:14:23-6.

7. Nofal WH, El Fawal SM, Shoukry AA, et al. Ultrasound-guided axillary brachial plexus block versus local infiltration anesthesia for arteriovenous fistula creation at the forearm for hemodialysis in patients with chronic renal failure. Saudi I Anaesth. 2017;11(1):77-82.

8. Kant A, Gupta PK, Zohar S, Chevret S, Hopkins PM. Application of the continual reassessment method to dose-finding studies in regional anesthesia: an estimate of the ED95 dose for 0.5\% bupivacaine for ultrasound-guided supraclavicular block. Anesthesiology. 2013;119(1):29-35.

9. Gupta PK, Pace NL, Hopkins PM. Effect of body mass index on the ED50 volume of bupivacaine $0.5 \%$ for supraclavicular brachial plexus block. Br J Anaesth. 2010;104(4):490-5. https://doi.org/10.1093/bja/aeq017. Epub 2010 Feb 18. PubMed PMID: 20,167,584

10. Bigeleisen PE, Moayeri N, Groen GJ. Extraneural versus intraneural stimulation thresholds during ultrasound-guided supraclavicular block. Anesthesiology. 2009;110(6):1235-43.

11. Fredrickson MJ, Smith KR, Wong AC. Importance of volume and concentration for ropivacaine interscalene block in preventing recovery room pain and minimizing motor block after shoulder surgery. Anesthesiology. 2010 Jun;112(6):1374-81.

12. Hermanns $H$, Braun $S$, Werdehausen $R$, Werner A, Lipfert P, Stevens MF. Skin temperature after interscalene brachial plexus blockade. Reg Anesth Pain Med. 2007 Nov-Dec;32(6):481-7.

13. Nakamura T, Popitz-Bergez F, Birknes J, Strichartz GR. The critical role of concentration for lidocaine block of periph- eral nerve in vivo: Studies of function and drug uptake in the rat. ANESTHESIOLOGY. 2003:99:1189-97.

14. Vandepitte C, Gautier P, Xu D, et al. Effective volume of ropivacaine $0.75 \%$ through a catheter required for interscalene brachial plexus blockade. Anesthesiology. 2013 Apr;118(4):863-7.

15. Lahaye LA, Butterworth JF 4th. Interscalene brachial plexus blocks under general anesthesia in adults. Reg Anesth Pain Med. 2015 May-Jun;40(3):293.

16. Li J, Karmakar MK, Li X, Kwok WH, Ngan Kee WD. Regional hemodynamic changes after an axillary brachial plexus block: a pulsed-wave Doppler ultrasound study. Regional Anesthesia and Pain Medicine. 2012;37:111-8.
Submit your next manuscript to BioMed Central and we will help you at every step:

- We accept pre-submission inquiries

- Our selector tool helps you to find the most relevant journal

- We provide round the clock customer support

- Convenient online submission

- Thorough peer review

- Inclusion in PubMed and all major indexing services

- Maximum visibility for your research

Submit your manuscript at www.biomedcentral.com/submit
C) BioMed Central 